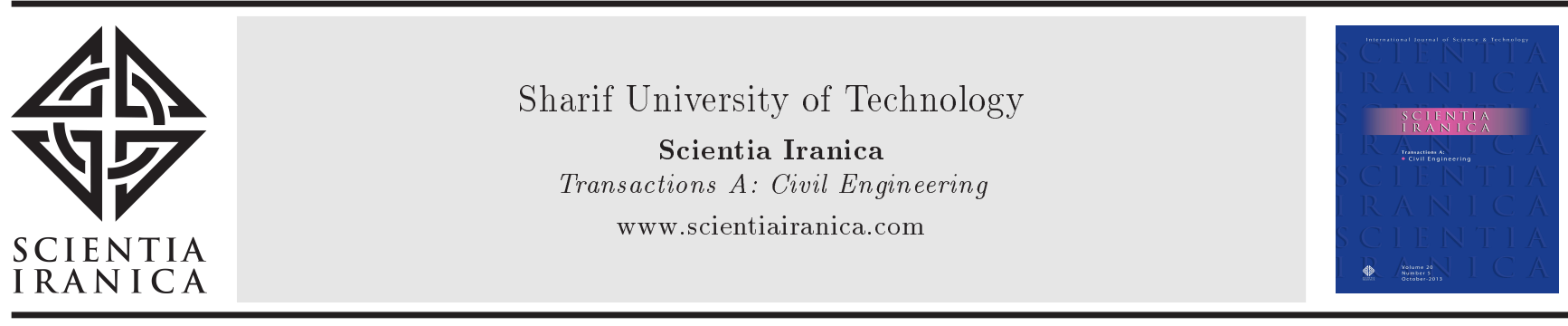

\title{
Uncertainty assessment of critical excavation depth of vertical unsupported cuts in undrained clay using random field theorem
}

\author{
R. Jamshidi Chenari* and M. Zamanzadeh \\ Department of Civil Engineering, Faculty of Engineering, University of Guilan, Rasht, Guilan, Iran.
}

Received 14 December 2014; received in revised form 22 May 2015; accepted 19 October 2015

\section{KEYWORDS \\ Critical excavation depth; \\ Probability of failure; \\ Spatial variability; \\ Random finite \\ difference method; \\ Random limit \\ equilibrium method.}

\begin{abstract}
Classical methods such as limit equilibrium or limit analysis, dealing with the stability analysis of open cuts and trenches and calculation of critical excavation depth for them, do not fully satisfy the theoretical requirements for stability problems leading to different solutions depending on the adopted method. It is now appreciated that geomaterials exhibit considerable heterogeneity, caused by the lithological and inherent variation, which cannot be fully covered by simple methods. This paper highlights the uncertainty embedded in critical excavation depth calculation, arising from spatial variability of shear strength parameters, using Random Finite Difference Method (RFDM) and Random Limit Equilibrium Method (RLEM). In the present study, the lognormally distributed undrained shear strength is considered spatially correlated throughout the domain. Surface cohesion value and the shear strength density were introduced as the deterministic parameters along with the coefficient of variation of undrained shear strength and its scale of fluctuation as stochastic parameters; these parameters were studied to see their effect on uncertainty in critical excavation depth estimation. The results clearly demonstrated the uncertainty in critical excavation depth arising from the inherent variability of shear strength parameters using the RFDM results; however, RLEM did not prove to reflect such uncertainty efficiently due to local averaging in prescribed failure surfaces.

(C) 2016 Sharif University of Technology. All rights reserved.
\end{abstract}

\section{Introduction}

Slope and trench stability analysis is a branch of geotechnical engineering that is highly adaptable to probabilistic treatment and has received considerable attention in the literature. Almost all practical probabilistic methods have, at some points, been applied to slope stability problems as a general earth struc-

*. Corresponding author. Tel.: +98 13-33690485; Fax: +98 13-33505866

E-mail addresses: Jamshidi_reza@guilan.ac.ir (R. Jamshidi Chenari); Masoud_zamanzadeh@yahoo.com ( $M$.

Zamanzadeh) ture analysis. A deterministic slope stability analysis method such as the Limit Equilibrium Method (LEM), Limit Analysis Method (LAM), or Finite Element/Difference Method (FEM/FDM) is needed as the basis of a probabilistic slope stability analysis. The choice of a deterministic slope stability analysis method also determines how spatial variability can be applied.

Since four decades ago, many probabilistic methods have been contrived for analysis of the stability of earth structures. These methods can be grouped into four categories: analytical methods, approximate methods, Monte-Carlo simulation, and finite element method. In analytical methods, the probability density functions of input variables are expressed mathemati- 
cally. They are then integrated analytically into the adopted slope stability analysis model to derive a mathematical expression for the density function of the factor of safety. Limited attempts have been made to utilize analytical methods [1-3]. The jointly distributed random variables method belongs to this category.

Most approximate methods are modified versions of two methods, i.e. First Order Second Moment (FOSM) Method [4] and Point Estimate Method [5]. Both methods require knowing the mean and variance of all input variables as well as the performance function defining safety factor (e.g., Bishop's equation). Some research on slope stability by the FOSM method is reported (e.g., [6]). Griffiths et al. [7] used random field theory in probabilistic analysis of infinite slopes and concluded that the first order methods may not properly account for spatial variability which can lead to unconservative estimates of the probability of slope failure. Some attempts have also been made to apply the Point Estimate Method [8-9].

A Monte-Carlo simulation is a procedure which seeks to simulate stochastic processes by random selection of input values to an analysis model in proportion to their joint probability density function. It is a powerful technique that is applicable to both linear and non-linear problems, but requires a large number of simulations to provide a reliable distribution of the responses. Many attempts have been made to analyze the stability of slopes using Monte-Carlo simulation [10$15]$.

The random finite element method combines elasto-plastic finite element analysis with random fields generated using the local average subdivision method. Several new slope stability analyses have been done using this method and the stochastic finite element method [16-21].

The current study investigates the statistical and probability issues involved in stability of vertical unsupported cut slopes in undrained condition employing two distinctively different reliability analysis methods, namely, Random Limit Equilibrium Method (RLEM) and random finite difference method (RFDM). Although necessity of support system for vertical cuts cannot be neglected, it should be clarified that this study does not consider any retaining system for vertical cuts. The aim of the study is only to show the effect of variability in shear strength parameters on the critical excavation depth estimation of unsupported vertical cuts.

\section{Spatial variability and reliability}

The spatial variability of geotechnical profiles can be quantified by using several statistical parameters, such as the central trend (or the mean), the coefficient of variation, the correlation length, and the anisotropy

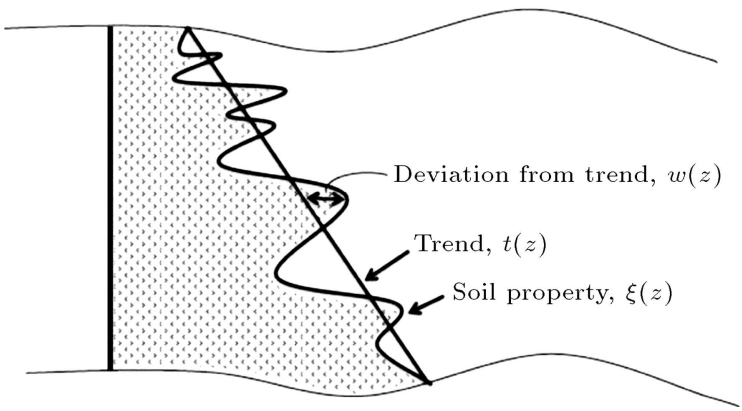

Figure 1. Inherent soil variability along with different components [22].

among others (e.g., [22-23]). For example, the spatial variation in geotechnical property, $\xi$, with depth $z$ can be decomposed into a trend function $t$ and a fluctuating component $w$ :

$$
\xi(z)=t(z)+w(z) .
$$

Figure 1 schematically represents different components of inherent variability. Deterministic trend can be estimated by a reasonable amount of in situ soil data (using for example least-square fit method), where the fluctuating component can be characterized as a random variable having zero mean and non-zero variance.

The standard deviation, $\sigma(z)$, normalized by the local mean geotechnical property, $\mu(z)$, obtained from the trend function provides a useful dimensionless ratio known as the Coefficient of Variation $(\mathrm{CoV})$ :

$$
\operatorname{CoV}(z)=\frac{\sigma(z)}{\mu(z)} .
$$

The generic range of CoVs for soil properties is summarized in Table 1. More comprehensive discussion on the $\mathrm{CoV}$ ranges and other statistical parameters for geotechnical properties are provided in Phoon and Kulhawy [24].

The scale of geotechnical property fluctuation is another important spatial characteristic of the ground. It indicates the distance scales within the material properties that show strong spatial correlations. The scale of fluctuations in geotechnical random fields can be simulated by using correlation lengths in covariance functions. The correlation length, or autocorrelation length, is the distance at which spatial autocorrelations

Table 1. Representative Coefficients of Variation (CoV) for geotechnical parameters [27].

\begin{tabular}{lc}
\hline Property & CoV (\%) \\
\hline Dry unit weight $\left(\gamma_{d}\right)$ & $2-13$ \\
Undrained shear strength $\left(C_{u}\right)$ & $6-80$ \\
Effective friction angle $\left(\varphi^{\prime}\right)$ & $7-20$ \\
Elastic modulus $\left(E_{s}\right)$ & $15-70$ \\
\hline
\end{tabular}


decay by $1 / e$, which is about $37 \%$ (e.g., [25]). The scale of fluctuations is usually between 1.4 and 2.0 times the correlation length for exponential, squared exponential, and spherical autocorrelation functions (e.g., [26]). The Markovian spatial correlation function is commonly used in geotechnical engineering to simulate the soil heterogeneity (e.g., [27]).

\section{Random Limit Equilibrium Method (RLEM)}

Although most traditional limit equilibrium methods do not consider spatial variability, some investigators have combined LEM with random field theory (e.g., [28-37]). However, the inherent nature of LEM is that it leads to a critical failure surface which in 2-D analysis appears as a straight line or curvilinear shape that could be noncircular. The influence of random field is only taken into account along a prescribed line or curvilinear path. Table 2 provides a list of 2-D slope stability reliability studies found in the literature utilizing LEM and random field theory in some cases.

The objective of this study is to show the effect of uncertainty embedded in geotechnical parameters (undrained cohesion in this study) on critical excavation depth estimation. It is quite impossible to find an analytical solution for critical excavation depth $\left(H_{c r}\right)$ while considering the log-spiral slip surface, and it demands fully-numerical methods to render a solution; therefore, a planar slip surface is defined in order to obtain critical excavation depth in a specific soil condition.

Denoting the specific weight of the soil body by $\gamma$, cohesion value at the ground surface by $C_{u 0}$, and the shear strength rate or density value by $\lambda$, according to the Limit Analysis Method (LAM), the equilibrium satisfaction leads to a general solution for the factor of safety of unsupported vertical cuts adopting a planar slip surface in undrained condition as:

$$
\text { F.S. }=\left[\frac{\alpha C_{u}}{H(\gamma-\beta \lambda)}\right] \text {. }
$$

$\alpha$ and $\beta$ values in Eq. (3) vary between 2 to 4 and 1 to 2 , respectively, depending on the adoption of lower or upper bound schemes. As will be discussed later, $\alpha$ and $\beta$ values can be precisely approximated by the fact that the value obtained by Eq. (3) should converge to that obtained by strength reduction method.

Practically, the geotechnical engineers use the planar or more advanced log-spiral slip surface to investigate the factor of safety of a vertical trench and consequently its critical excavation depth. In LEM analyses, it is not required to consider realizations in the whole body of the problem and thus the very timeconsuming factor of safety calculation process for each realization is averted; also, it seems very simple to take account of just the slip surface over which realization can be done. To do this, it is first needed to identify the slip surface and then the slip surface can be discretized and divided into elements having correlated cohesion values. Two approaches are available here: the planar slip surface and the log-spiral one. In the undrained condition for the planar slip surface, slip angle would be $\pi / 4$ with respect to the vertical plane. As stated in literature [43], the slip surface is not planar; hence, for the undrained condition, the log-spiral slip surface formula is also employed, which would be transformed to the circular one for undrained condition.

Before conducting any stochastic analysis, model geometry was adopted corresponding to unit safety factor for each set of geotechnical parameters. Although the retaining structures are mandatory to

Table 2. Slope stability reliability studies combining LEM with random field theory in some cases.

\begin{tabular}{lll}
\hline Deterministic method & \multicolumn{1}{c}{ Probabilistic method } & \multicolumn{1}{c}{ Reference } \\
\hline Level-crossing method & First Order Second Moment (FOSM) & Catalan and Cornell [38] \\
Bishop & First Order Second Moment (FOSM) & Alonso [39] \\
Morgenstern-Price & First Order Reliability Method (FORM) & Li and Lumb [28] \\
Morgenstern-Price & First Order Reliability Method (FORM) & Mostyn and Soo [29] \\
Bishop & Monte-Carlo Simulation (MCS) & El-Ramly et al. [32] \\
Spence & First Order Reliability Method (FORM) & Low [33] \\
Bishop & First Order Second Moment (FOSM) & Sivakumar et al. [34] \\
LEM & Monte-Carlo Simulation (MCS) & Cho [36] \\
Spencer & First Order Reliability Method (FORM) & Low et al. [35] \\
Chen and Morgenstern & Monte-Carlo Simulation (MCS) & Hong and Roh [6] \\
LEM & Importance sampling technique & Ching et al. [40] \\
LEM & Monte-Carlo Simulation (MCS) & Ching et al. [41] \\
LEM & Kriging-based response surface method & Zhang J. et al. [42] \\
\hline
\end{tabular}


be utilized in such cases, the aim of this study is only to show the effect of spatial variability of shear strength parameters on stability of vertical cuts when a small variability probably leads to more probability of failure in comparison with the classic deterministic cases. Uncertainty was applied to the undrained shear strength so as to assess the reliability of calculations by rendering a safety factor for each stochastic realization. For this reason, two approaches were adopted. The first approach considers planar slip surface and divides it into 1000 elements. The second approach considers circular slip surface and seeks the most critical circular slip surface as traditionally adopted in slope stability analysis literature. In the first approach, for the planar slip surface, the plane of slip is divided into 1000 elements and an auto-correlated cohesion field using random field theory is generated to assign the related cohesion to each element. In the second approach, a mesh grid has been generated while each grid point represents the center of a circular slip surface with a specified radius, as shown in Figure 2. Several slip surfaces can be found; however, the best of them corresponds to the minimum deterministic safety factor sought based on a simple optimization scheme found in conventional slope stability analyses. After selection of the critical slip surface, as schematically illustrated in Figure 2, 1000 elements can be created along it and realizations can be performed to set a combination of auto-correlated cohesion values afterwards.

Using Bishop's formula to calculate the factor of safety of a circular slip surface and assuming that each slice has its own cohesion value obtained by random field theory, the safety factor can be calculated by:

$$
\text { Safety factor }=\frac{\sum_{i=1}^{n} C_{\text {Realized }} \cdot b_{i} / \cos \theta_{i}}{\sum_{i=1}^{n} w_{i} \cdot \sin \theta_{i}},
$$

in which $b_{i}$ is the horizontal width of each slice (project length of each element on the horizon), $\theta_{i}$ is the inclination angle for each slice, and $w_{i}$ is the weight of the respective slice. $C_{\text {Realized }}$ can be obtained by

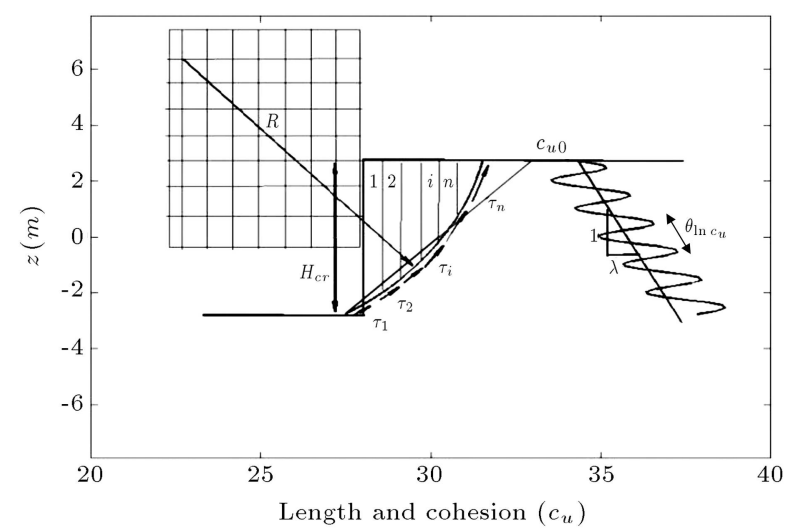

Figure 2. Finding the best estimated circular slip surface based on Bishop's formulation.

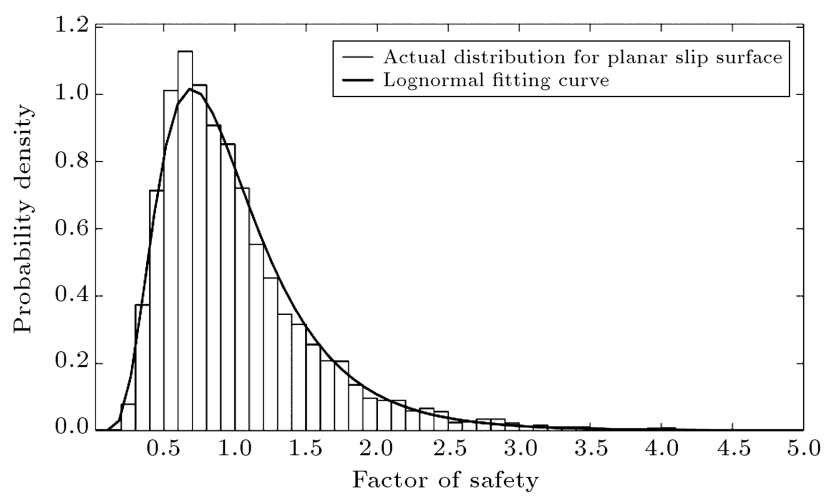

(a)

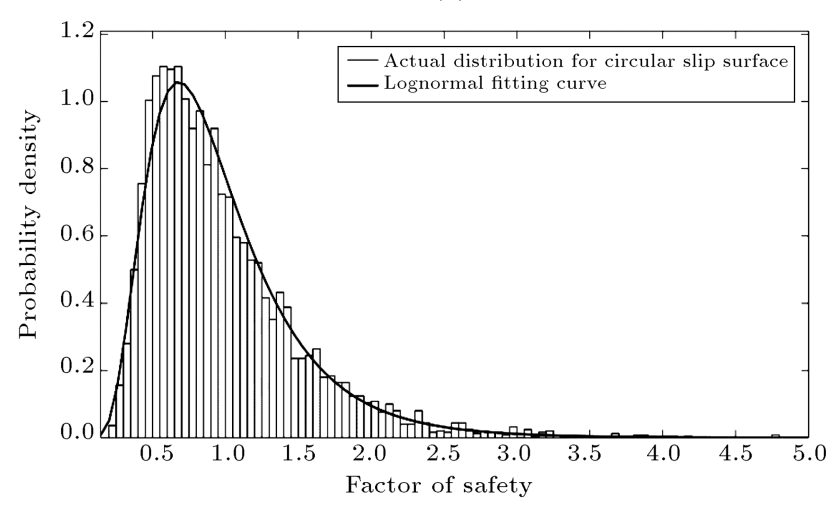

(b)

Figure 3. Best fitted curve on the result of RLEM with $C_{u 0}=50 \mathrm{kPa}, \lambda=1.0 \mathrm{kPa} / \mathrm{m}, \mathrm{CoV}_{C u}=50 \%$, and $\theta=24 \mathrm{~m}$ : (a) Planar slip surface; and (b) circular slip surface.

the result of Monte-Carlo realizations as discussed earlier. For both planar and circular slip surface, 1000 realizations have been performed. It is observed from Figure 3 that the results of safety factor calculation for both failure slip assumptions follow a lognormal Probability Distribution Function (PDF).

\section{Random Finite Difference Method (RFDM)}

In recent years, a more rigorous method of probabilistic geotechnical analysis has been pursued in which nonlinear finite element/difference methods are combined with random field generation techniques (e.g., [16-17]). This method, called the "Random Finite Element/Difference Method" (RFEM/RFDM), fully accounts for spatial correlation and averaging and is also a powerful slope stability analysis tool that does not require a priori assumptions related to the shape or location of the failure mechanism. To calculate the factor of safety, the C-Phi reduction approach was employed in which the strength characteristics of soil are reduced to where a failure occurs. The nonlinear finite difference program FLAC [44], which takes the mechanical behavior of excavations into account and is able to calculate the factor of safety of slopes, 


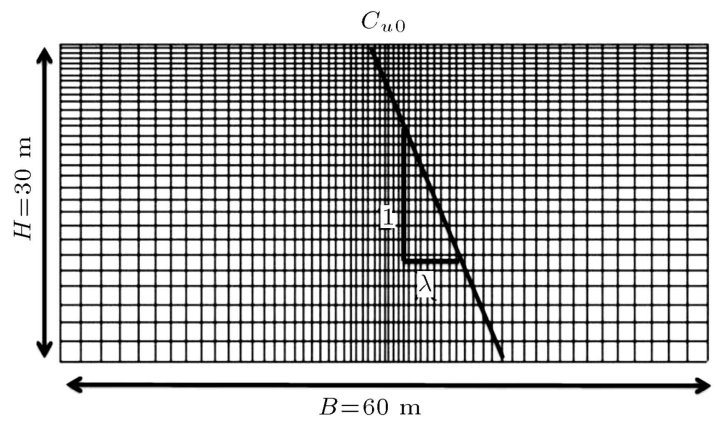

Figure 4. Configuration of the model utilized in the analysis.

was utilized to analyze the stability of unsupported trenches. Typical geometry of the model analyzed in this study is shown in Figure 4.

The finite difference mesh has been examined to eliminate the influence of size and boundary effect on the accuracy of results. Analyses with deterministic values of undrained shear strength increasing with depth were first performed to see the accuracy of the proposed method and compare it with the existing approximate solutions. The undrained Young's modulus is defined as $500 C_{u}$ increasing with depth, accordingly. In the C-Phi reduction-based approach, the step by step excavation proceeds until a safety factor of 1.0 is approached. Based on this method, the deterministic critical excavation depth for different surface cohesion values is seen to be higher than the lower bound solution values and lower than the upper bound solution values as shown in Figure 5 . The calculated deterministic critical excavation depth corresponding to zero coefficient of variation is then employed in subsequent stochastic analyses so as to apply uncertainty by introducing stochastic variation to the deterministic trend. The objective of this study is to show the effect of uncertainty embedded in geotechnical parameters (undrained cohesion in this study) on critical excavation depth estimation.

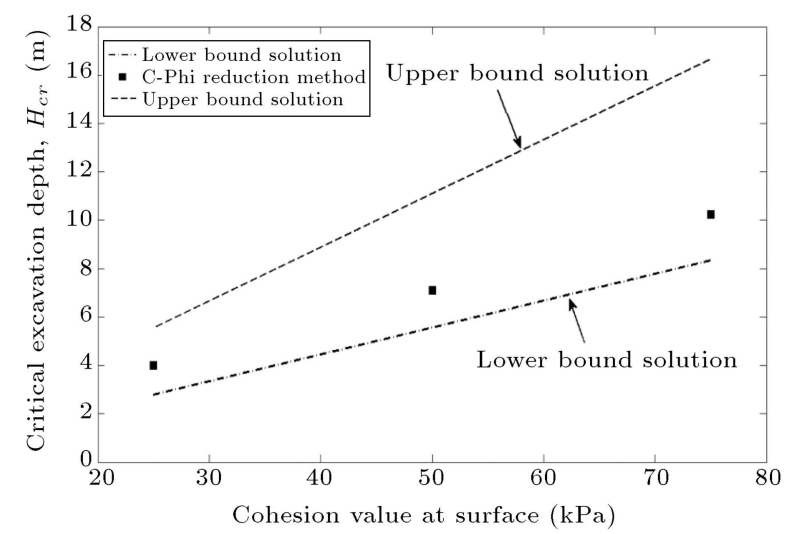

Figure 5. The critical excavation depth calculated from deterministic strength reduction analysis for different surface cohesion values with $\lambda=1.0 \mathrm{kPa} / \mathrm{m}$.
Obviously, the starting point was chosen based on a deterministic C-Phi reduction analysis assuming "zero" coefficient of variation corresponding to a "unit" safety factor. Spatial variability of shear strength parameters was then applied to the domain under study to see its effect on the reliability of deterministic analysis schemes. Even a small variation in shear strength $(\mathrm{CoV}=10 \%)$ might lead to a great probability of failure, which is expected while the deterministic factor of safety is taken as "unity". The aim of the study is only to compare the probabilities of failure $\left(P_{f}\right)$ corresponding to different Coefficients of Variation (CoVs). Therefore, the absolute values of $P_{f}$ are not of value. Although retaining structures like cantilver retaining walls are mandatory to utilize in such cases, the study emphasizes the necessity of such provisions more than the deterministic cases when a small variability will lead to more probability of failure in comparison with the classic deterministic cases.

In the probabilistic studies, the stochastic variation of undrained shear strength is modeled by adopting a lognormal distribution with the aid of the most recognized representative statistical parameters including the mean value, the standard deviation, and the scale of fluctuation. There are several distributions like normal, lognormal, beta, etc. used in practice. However, the use of lognormal distribution lies in the fact that shear strength is strictly non-negative and in lognormal distribution, there is no possibility of the existence of negative values. For a detailed description of proper distributions in geomaterials, Lee et al. [45] and Harr [46] can be conferred. In practice, it is more common to use the dimensionless coefficient of variation instead of standard deviation, which can be defined as the standard deviation divided by the mean. Typical values for the $\mathrm{CoV}$ of the undrained shear strength have been suggested by several investigators based on in situ or laboratory tests as pointed out earlier. The third important feature of a random field is its correlation structure. It is obvious that if two samples are close together, they will usually be more correlated than the case where they are widely separated. It is common in literature to use a single exponential form of correlation function, known as Markovain spatial-correlation function:

$$
\rho=e^{\left(-\frac{2|\tau|}{\theta}\right)}
$$

in which $\theta$ is the scale of fluctuation, which is twice the correlation length for Markovian correlation function definition, and $\tau$ is the separation or lag distance. The correlation length is the parameter which describes the degree of correlation of a soil property and is defined as the distance beyond which the random values will be no more correlated at all. It should be noted that in the case of a small correlation length, random field 
tends to be rough, and, oppositely, when it is large, random field tends to be smooth [47].

If the undrained shear strength, $c_{u}$, is considered a random variable and assumed to be lognormally distributed throughout the domain of study, the point statistics parameters, i.e. mean, $\mu$; standard deviation, $\sigma$; and the most representative spatial statistics parameter, i.e. scale of fluctuation $\theta$, are used to realize a log-normally distributed random field given by:

$$
c_{u}(\tilde{x})=\exp \left(L \cdot \varepsilon_{\ln c_{u}(\tilde{x})}+\mu_{\ln c_{u}(\tilde{x})}\right)+\lambda z,
$$

where $c_{u}(\tilde{x})$ is the undrained shear strength assigned to the zones, $\tilde{x}$ is the spatial position at which $c_{u}$ is desired, $\varepsilon_{\ln c_{u}(\tilde{x})}$ is an independent normally distributed random field with zero mean and unit variance, $\mu_{\ln c_{u}}$ is the mean of the logarithm of $c_{u}, \lambda$ is the shear strength rate or density value as defined earlier, and $L$ is a lowertriangular matrix computed from decomposition of the covariance matrix.

$$
A=L L^{T}
$$

where $A$ is the covariance between the logarithms of the undrained shear strength values at any two points. In this study, $\ln C_{u}$ values are assumed to be characterized by an exponential Markovian covariance function given by [48]:

$$
A=\sigma_{\ln c_{u}}^{2} \times e^{\left(-\frac{2|\tau|}{\theta}\right)},
$$

where $\sigma_{\ln C_{u}}$ is the standard deviation of the logarithm of $c_{u}$, and $\tau$ is the lag distance, as mentioned before, considered to be the center to center distance of the consecutive zones.

The parameters $\mu_{\ln C_{u}}$ and $\sigma_{\ln C_{u}}$ are obtained from the specified mean and variance of undrained cohesion, using log-normal distribution transformations given by:

$$
\begin{aligned}
& \mu_{\ln c_{u}}=\ln \left(\mu_{c_{u}}\right)-\frac{1}{2} \sigma_{\ln c_{u}}^{2}, \\
& \sigma_{\ln c_{u}}^{2}=\ln \left(1+\left(\sigma_{c_{u}} / \mu_{c_{u}}\right)^{2}\right)=\ln \left(1+\mathrm{CoV}_{c_{u}}^{2}\right) .
\end{aligned}
$$

Once the covariance matrix is established, it is decomposed into lower and upper triangular matrices (Eq. (7)) using Cholesky decomposition technique. Then, realizations of lognormally distributed soil properties at each zone center are obtained by the transformation presented in Eq. (6) for a specified mean, standard deviation, and scale of fluctuation of the given soil property $\left(c_{u}\right)$.

Figure 6 demonstrates a flowchart on how the calculation proceeds and the step by step procedure for safety factor prediction is demonstrated. Similar procedure was adopted for approximate methods (LEM or LAM) with the sole difference on the method of safety factor calculation which is conceptually different from FDM. Figure 7 illustrates a sample realization

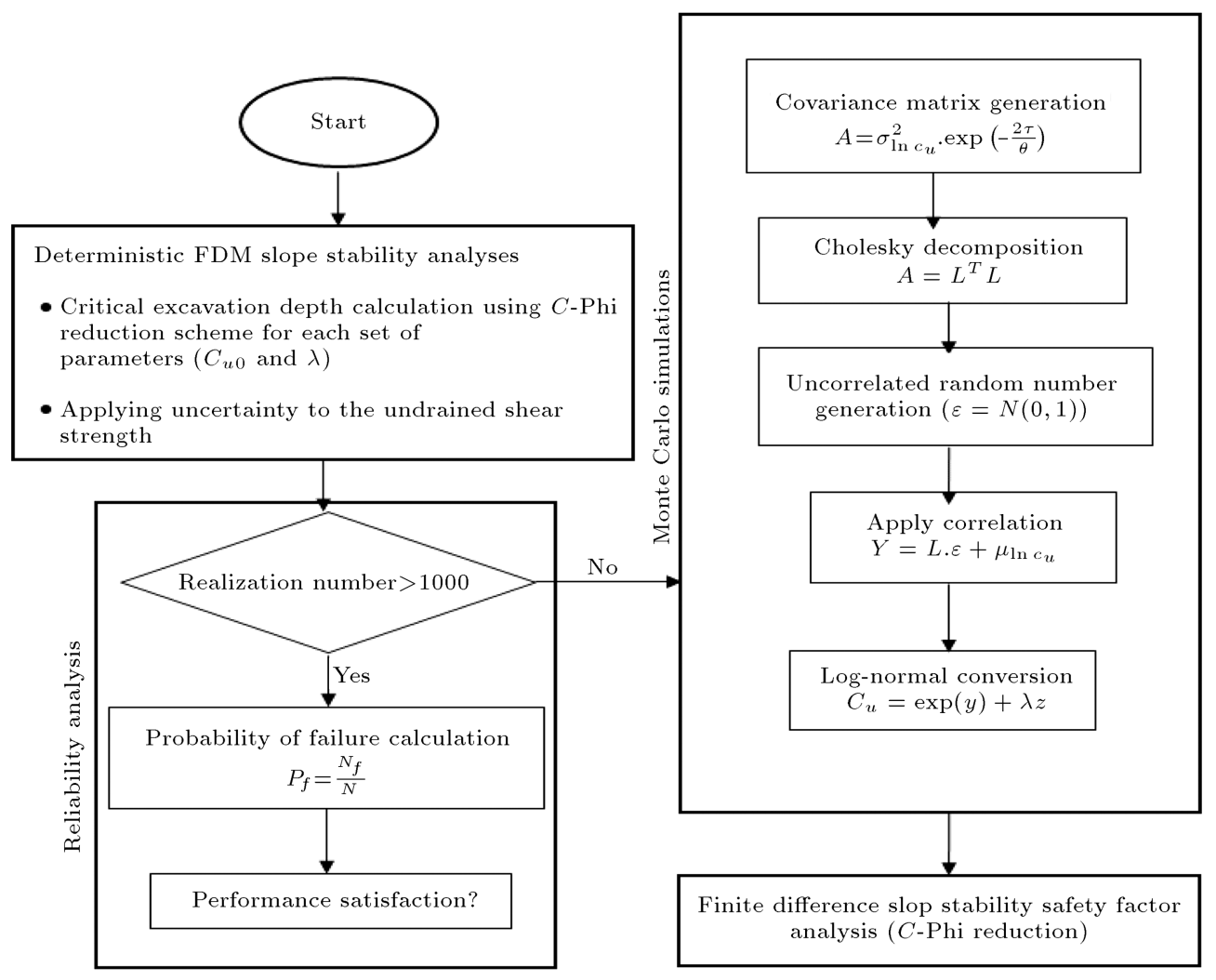

Figure 6. Flowchart for the process of safety factor analysis using RFDM. 


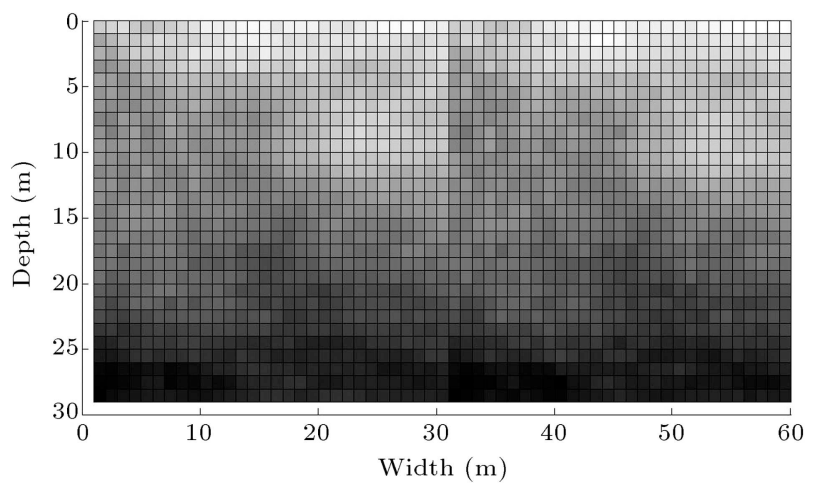

Figure 7. A typical realization of undrained shear strength with $C_{u 0}=50 \mathrm{kPa}, \lambda=1.0 \mathrm{kPa} / \mathrm{m}$, $\mathrm{CoV}_{C u}=10 \%$, and $\theta=200 \mathrm{~m}$.

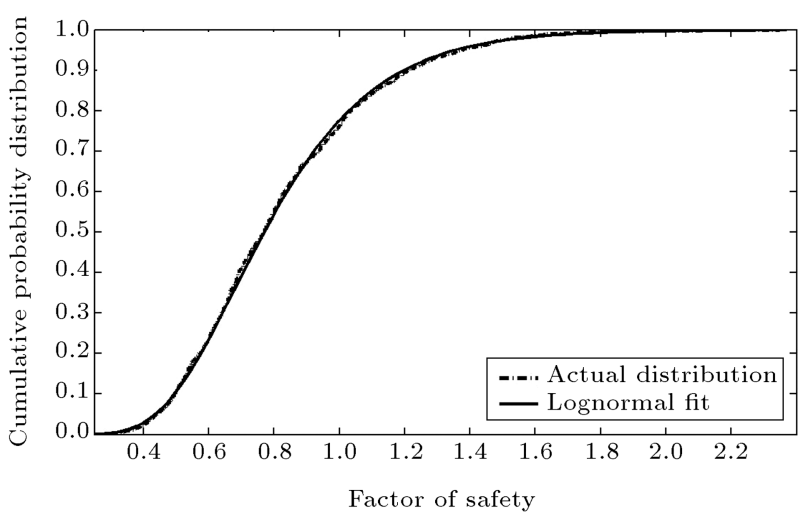

Figure 8. Calculated and fitted log-normal cumulative probability of the factor of safety for $C_{u 0}=50 \mathrm{kPa}$, $\lambda=1.0 \mathrm{kPa} / \mathrm{m}, \theta=24 \mathrm{~m}$, and $\mathrm{CoV}_{C u}=70 \%$.

of the undrained shear strength with the assumed stochastic properties.

A typical cumulative probability of the factor of safety, as estimated from 1000 realizations, is shown in Figure 8 for $C_{u 0}=50 \mathrm{kPa}$ and $\lambda=1 \mathrm{kPa} / \mathrm{m}$. A lognormal distribution was adopted in this study with the requirement that safety factor is non-negative. Superimposed on the cumulative probability is a fitted lognormal function with parameters given by $m_{\mathrm{FS}}=$ $0.817, \sigma_{\mathrm{FS}}=0.0844$. At least visually, the lognormal cumulative graph appears perfectly fitted.

\section{Results and discussion}

In RFDM analyses, the Poisson's ratio $(v)$ is assumed to be constant while the undrained shear strength $\left(C_{u}\right)$ and the Young's modulus $\left(E_{u}\right)$ are randomized throughout the domain. Forty sets of Monte-Carlo simulations were performed to investigate the effects of the surface cohesion, $C_{u 0}$, and undrained shear strength density, $\lambda$, as deterministic parameters and coefficient of variation of undrained shear strength, $\mathrm{CoV}_{C u}$, and its scale of fluctuation, $\theta$, representing stochastic parameters on reliability of an open cut exca-
Table 3. Different parameter sets adopted in this study.

\begin{tabular}{cc}
\hline Parameter & Considered values \\
\hline$C_{u 0}(\mathrm{kPa})$ & 50 \\
$\lambda(\mathrm{kPa} / \mathrm{m})$ & 1,2 \\
$C O V_{C u}(\%)$ & $10,30,50,70,90$ \\
$\theta(\mathrm{m})$ & $0.2,8,24,200$ \\
\hline
\end{tabular}

vation. The above-mentioned parameters are variable through different sets of analyses according to Table 3 .

Spatial correlation lengths in both the horizontal and vertical directions were held the same implying isotropic correlation structure. For the given problem configuration and element size, $\theta=0.2 \mathrm{~m}$ gives no appreciable correlation for the parameter under study and results in ragged property field. On the contrary, the length of $200 \mathrm{~m}$ represents the strict correlation between zones. For each set of adopted $C_{u 0}, \lambda, C O V_{C u}$, and $\theta$, Monte-Carlo simulations have been conducted involving 1000 realizations of the undrained shear strength random field and the subsequent numerical progress of calculating the factor of safety.

It is reasonable to estimate the probability of failure based on lognormal probability distribution according to the goodness-of-fit test. For example, in Figure 8, the probability of factor of safety falling less than unity gives:

$$
P_{f}=P[\mathrm{FS}<1]=\varphi_{\text {Longnormal }}\left(\frac{1-\mu_{\mathrm{FS}}}{\sigma_{\mathrm{FS}}}\right) \cong 0.7758 \text {. }
$$

Another approach to conclude about the probability of failure of the system is to estimate the number of cases corresponding to the factor of safety less than unity and then to estimate the probability of failure from Eq. (14) which renders similar results with good precision:

$$
P_{f}=\frac{N_{f}}{N}=0.7620,
$$

where $N_{f}$ is the number of failed cases and $N$ is the total number of cases. This again confirms the suitability of lognormal distribution function for the factor of safety data. The results show that the difference between two methods is less than $2 \%$.

The probability of failure shall mathematically increase with the coefficient of variation of cohesion values, as confirmed by Figure 9 . This can be explained by the essence of strength reduction method in which some weak points may cause instability to occur. The number of weak points soars with the increase in the coefficient of variation of cohesion. Figure 9 shows the probability of failure (probability of the factor of safety falling below the unity) for different strength densities computed by RFDM analyses. The rate of increase in the probability of failure with 


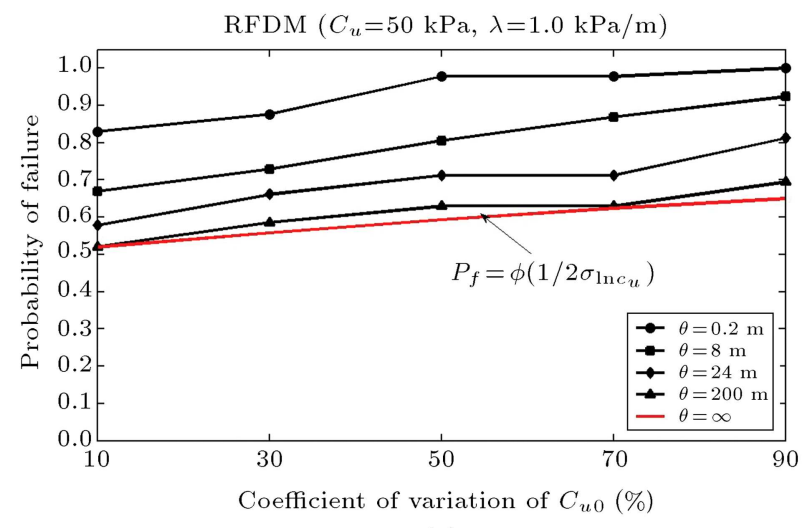

(a)

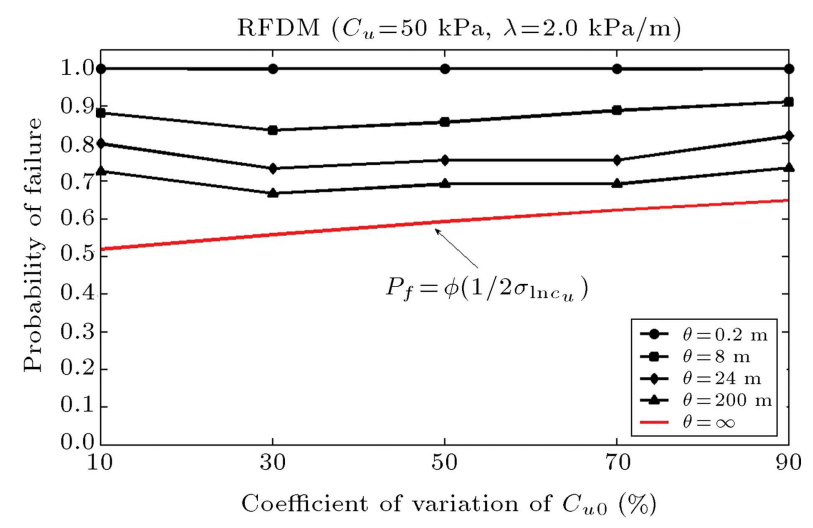

(b)

Figure 9. The probability of failure computed by RFDM analyses versus the coefficient of variation of undrained shear strength for $C_{u 0}=50 \mathrm{kPa}$ : (a) $\lambda=1.0 \mathrm{kPa} / \mathrm{m}$; and (b) $\lambda=2.0 \mathrm{kPa} / \mathrm{m}$.

$\mathrm{CoV}_{c u}$ variation however decreases when increasing the strength density $(\lambda)$. This is clear from the comparison of different parts in Figure 9 and it is mainly due to the fact that when the undrained shear strength density $(\lambda)$ increases, the deterministic portion of the cohesion field gets more highlighted than the stochastic component and so the effect of stochastic variation becomes less important. This is endorsed by the fact that the coefficient of variation is defined based on the surface cohesion value, $C_{u 0}$, not the whole deterministic trend, and indeed increasing the strength density translates into decreased coefficient of variation with depth.

To investigate the effect of the scale of fluctuation, $\theta_{\ln C_{u}}$, on the safety factor statistics, $\theta_{\ln C_{u}}$ is varied from 0.2 (i.e., very much smaller than the soil model size) to 200 (i.e., very much bigger than the soil model size). In the limit, smaller values of $\theta_{\ln C_{u}}$ than the element size result in a set of elements which are largely independent (increasingly independent as $\theta_{\ln C_{u}}$ decreases) and the undrained shear strength field becomes a white noise, with independent $C_{u}$ values at any two distinct points. In such cases, the undrained shear strength field becomes infinitely "rough" and any point at which the soil is weak will be surrounded by points where the soil is strong. A path through the weakest points in the soil might have very low average strength, but at the same time, it will become infinitely tortuous and thus infinitely long. If the failure criterion was to seek a Critical Slip Line (CSL) bearing the minimum shear strength or contouring plastic points, the above-mentioned effects, combined with shear interlocking dictated by stress field, would imply that the weakest path should return to the traditional log-spiral (circular in undrained state) as explained by Fenton and Griffiths [49]. However, the routine safety factor calculation scheme adopted in FLAC is the socalled strength reduction method which seeks to find nonconvergent point(s) being an indicator of collapse. When the algorithm cannot converge within a userspecified maximum number of iterations, together with a dramatic increase in nodal displacements within the mesh, the implication is that no stress distribution can be found that is able to simultaneously satisfy both the failure criterion and global equilibrium, and failure is said to have occurred. The aforesaid failure criterion implies that weak point(s) formation may lead to the early nonconvergence and the failure state in other words. Therefore, the scale of fluctuation has a serious effect on the factor of safety as any decrease in the scale of fluctuation leads to a reduction in safety factor or an increase in probability of failure, as appears from Figure 9.

As $\theta_{\ln C_{u}} \rightarrow \infty$, the stochastic component of undrained shear strength field becomes the same everywhere. In this case, the factors of safety statistics are expected to approach those obtained by using a linearly varying lognormally distributed variable, $C_{u}$, to model the soil, $C_{u}(x)=C+\lambda z$. That is because the safety factor, FS, of a vertical trench in a soil layer with linear (but random) undrained shear strength, $C_{u}$, is given by:

$$
\mathrm{FS}=\frac{\mathrm{FS}_{d e t}}{\mu_{c_{u}}} c_{u},
$$

where $\mathrm{FS}_{\text {det }}$ is the "deterministic" safety factor when $C_{u}=C_{u 0}+\lambda z$, and which is obtained from a single finite difference analysis (or any other appropriate approximate calculation); then, as $\theta_{\ln C_{u}} \rightarrow \infty$, the safety factor assumes a lognormal distribution with parameters:

$$
\begin{aligned}
\mu_{\ln \mathrm{FS}} & =\ln \mathrm{FS}_{d e t}+\ln \mu_{c_{u}}-\mu_{\ln \mu c_{u}}=-\frac{1}{2} \sigma_{\ln c_{u}}^{2}, \\
\sigma_{\ln \mathrm{FS}} & =\sigma_{\ln c_{u}},
\end{aligned}
$$

where $\mathrm{FS}_{\text {det }}$ is taken as unity in Eq. (15), as discussed earlier, and the relationships for the parameters of transformed $\ln C_{u}$ Gaussian random field are employed. The probability of failure can be calculated assuming 
a lognormal distribution for the safety factor.

$$
P_{f}=\varphi\left(-\frac{\mu_{\operatorname{ln~FS}}}{\sigma_{\operatorname{ln~FS}}}\right)=\varphi\left(\frac{1}{2} \sigma_{\ln c_{u}}\right),
$$

Figure 9 suggests that the probability of failure of the vertical cut with largely correlated undrained shear strength field can be approximately estimated by a straight line of the form suggested by Eq. (16). It can be seen that the agreement is fairly acceptable. The small deviation arises from the fact that the predicted probability of failure computed from Eq. (18) is based on a LEM assumption (Eq. (15)) which takes a planar slip surface into account for a model bearing linearly depth-varying undrained shear strength property, and the FDM solution which adopts the strength reduction scheme to seek a failure point is conceptually different and thus a deviation might be expected.

Another important stochastic property of interest for the factor of safety is the coefficient of variation of the safety factor. Figure 10 illustrates the variation of the safety factor against the $\mathrm{CoV}$ of undrained shear strength. It is noted that introducing variation in cohesion field causes variability of the results and the $\mathrm{CoV}_{\mathrm{FS}}$ increases with increase in the $C O V_{C u}$. However, this behavior becomes less eminent when increasing the strength density from $\lambda=1 \mathrm{kPa} / \mathrm{m}$ to $\lambda=2 \mathrm{kPa} / \mathrm{m}$.

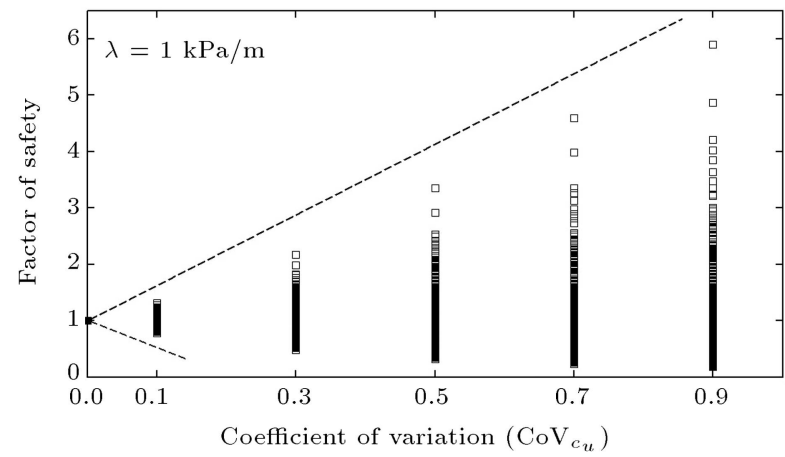

(a)

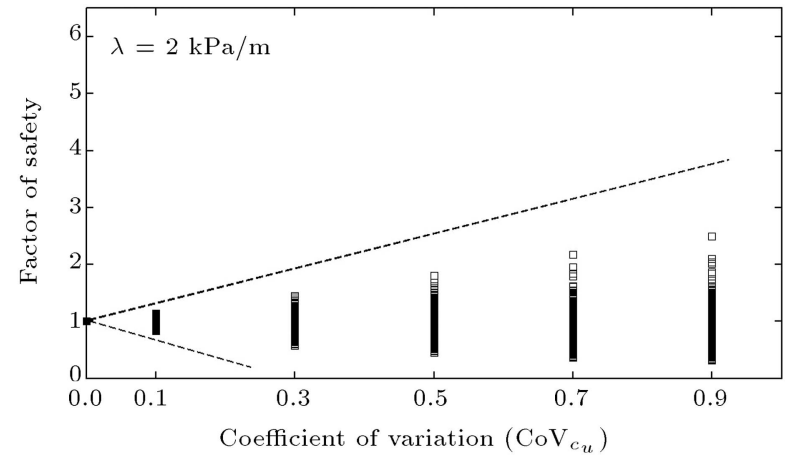

(b)

Figure 10. Variability of the factor of safety versus the coefficient of variation of undrained shear strength computed by RFDM analyses for $C_{u 0}=50 \mathrm{kPa}$ : (a) $\lambda=1.0 \mathrm{kPa} / \mathrm{m}$; and (b) $\lambda=2.0 \mathrm{kPa} / \mathrm{m}$.
This is clearly seen from comparing different parts of Figure 10. It is again explained by resorting to the fact that the increase in strength density leads the deterministic component of cohesion to have more important role than the stochastic component, as discussed earlier.

The RFDM is more time-intensive than the RLEM analyses which seek failure only along a prescribed and plausible failure surface. As RLEM analyses take place along a prescribed surface, one may look into the effects in a different view. The affecting parameters, namely the coefficient of variation and the scale of fluctuation of undrained shear strength, are studied while assuming a planar or circular slip surface. Figure 11 demonstrates the variation of the probability of failure of slope as defined earlier with the variability of undrained shear strength for two different strength densities. All scales of fluctuation are drawn in the plot and individually labeled. It is implied in these plots that the probability of failure of the vertical cut is not largely dependent on the scale of fluctuation, $\theta_{\ln C_{u}}$. This is as expected since the scale of fluctuation does not affect the global average of a normally distributed process along a predefined slip surface. The shear resistance along a plausible

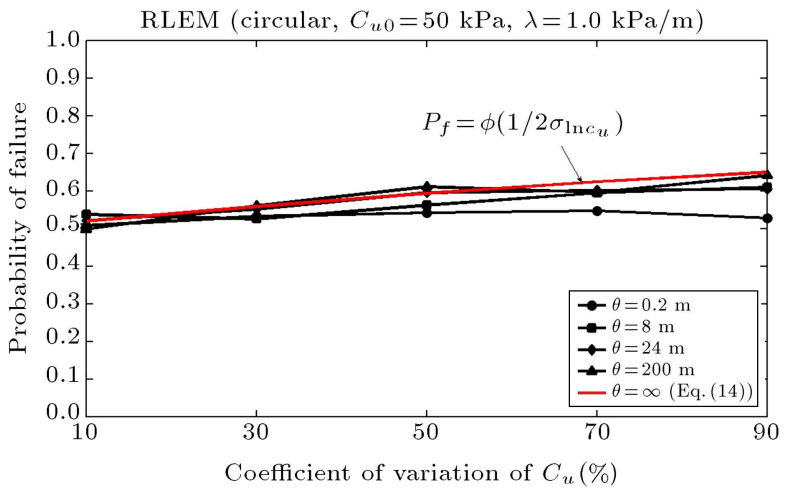

(a)

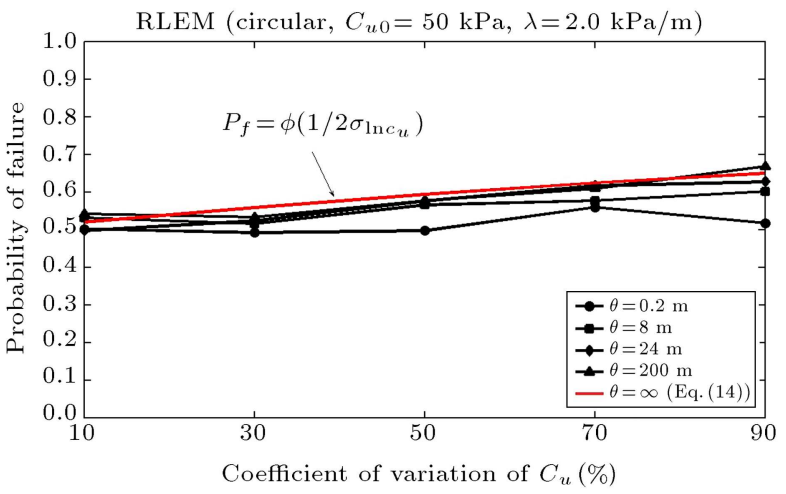

(b)

Figure 11. The probability of failure computed by RLEM analyses versus the coefficient of variation of undrained shear strength assuming a circular slip surface for $C_{u 0}=50 \mathrm{kPa}:$ (a) $\lambda=1.0 \mathrm{kPa} / \mathrm{m}$; and (b) $\lambda=2.0 \mathrm{kPa} / \mathrm{m}$. 
and predefined slip surface is computed by global integration of the undrained shear strength along a circular surface and it is expected not to be affected by the correlation structure of the random process. Figure 11 suggests that the probability of failure of vertical cut in an undrained condition computed by RLEM analyses can be approximately estimated by a straight line defined by Eq. (16).

\section{Conclusion}

The main purpose of the study was to evaluate the reliability of the calculated critical excavation depth considering spatially variable random shear strength field. The factor of safety of excavation in a spatially variable layer of finite depth overlying bedrock is well represented by a lognormal distribution with parameters $\mu_{\ln F S}$ and $\sigma_{\ln F S}$, while the affecting strength parameter (' $C_{u}$ ' in this study) is also assumed to be lognormally distributed.

Random Finite Difference Methods (RFDM) coupled with strength reduction method have been adopted to calculate the factor of safety in a spatially correlated random field. Two deterministic parameters, i.e. surface cohesion $\left(C_{u 0}\right)$ and the undrained shear strength rate $(\lambda)$, along with two stochastic parameters, i.e. the coefficient of variation of shear strength $(C O V)$ and its scale of fluctuation $(\theta)$, have been considered to evaluate the effect of the spatial variation of the input parameter on the uncertainty embedded in calculation of the critical excavation depth.

Results show that increasing the coefficient of variation of undrained cohesion leads to more probability of failure or uncertainty in other words. It is seen that even $10 \%$ variation in undrained shear strength may lead to unsatisfactory performance. Furthermore, it is observed that the more the undrained shear strength varies, the more scattering in safety factor estimation is expected. Scale of fluctuation of the undrained shear strength, on the other hand, shows a different effect as it causes the probability of failure to rise when decreasing the correlation between shear strength data. The explanation of this observation is inspired by the fact that in low values of the scale of fluctuation, zones become highly independent and a socalled "Ragged" field forms. This causes an increase in the number of weak points in the whole body, which in effect induces a reduction in the factor of safety due to the essence of strength reduction method. Strength density of the cohesion value, $\lambda$, on the other side of the spectrum, has a decreasing effect on the uncertainty of results owing to the fact that the coefficient of variation $(C O V)$ has been defined only based on the surface cohesion value $\left(C_{u 0}\right)$, and the increase in shear strength rate implies a reduction in coefficient of variation of undrained shear strength.
Random Limit Equilibrium Method (RLEM) was also adopted by choosing a planar and circular critical slip surface using Bishop's formula to investigate the effect of stochastic variation of undrained shear strength properties. The slip surface was then discretized into 1000 elements and an auto-correlated cohesion field was generated afterwards. The factor of safety for each realization was calculated using Bishop's formula. The results consistently demonstrated that the RLEM might not properly reflect the uncertainty in critical excavation depth estimation if the failure surface remained unchanged through realizations. The calculation of the safety factor in such simple and approximate methods was based mainly on the assumption of a plausible and predetermined slip surface. The shear resistance along the assumed failure surface was calculated by integration of the shear strength increments along the planar or circular surface and it was assumed not to be affected by the correlation structure of the random field and has vanishing variance for finite $\sigma_{\ln C_{u}}$. An important point is that in this study, RLEM first assumed the most probable slip surface corresponding to the deterministic condition and then applied uncertainty and variability of parameters across the same surface and did not change the surface location. However, it was possible to seek surface variation through realizations; but, if we are to investigate the heterogeneity effect by RLEM when considering critical failure mechanism variation, which is time-intensive, it is better to resort to RFDM or RFEM which render the most probable failure surface that passes through the weakest points and do not need any a priori assumptions regarding the shape and mechanism of the failure. Also, the potential pitfall of the LEM, which is the inability to account for the stress-strain behavior of the soil, is overcome.

\section{References}

1. McMahon, B.K. "Probability of failure and expected volume of failure in high rock slope", Australian New Zealand Conference on Geotechnics, pp. 308-313, Brisbane (1975).

2. Tobutt, D.C. and Richards, E.A. "The reliability of earth slopes", International Journal for Numerical Analytical Methods in Geomechanics, 3(4), pp. 323354 (1979).

3. Marek, J.M. and James P. "Probabilistic analysis on the planar shear failure mode", 19th U.S. Symposium on Rock Mechanics, Reno, Nevada, USA, pp. 40-44 (1978).

4. Ang, A.H. and Tang, W. "Probability concepts in engineering planning and design", 1, New York, USA: John Wiley and Sons (1984).

5. Rosenblueth, E. "Point estimates for probability moments", Proceeding of the National Academy of Science, 72(10), pp. 3812-3814, USA (1975). 
6. Hong, H.P. and Roh. G. "Reliability evaluation of earth slopes", Journal of Geotechnical and Geoenvironmental Engineering (ASCE), 134(12), pp. 1700-1705 (2008).

7. Griffiths, D.V., Huang, J. and Fenton, G.A. "Probabilistic infinite slope analysis", Computer and Geotechnics, 38(4), pp. 577-584 (2011).

8. Li, K.S. "A point estimate method for calculating the reliability index of slope", 6th Australian-New Zealand Conference on Geomechanics, pp. 448-451, Christchurch (1992).

9. Thornton, S. "Probability calculation for slope stability", Computer Methods and Advances in Geomechanics, pp. 2505-2509 (1994).

10. Chen, Q. and Chang, L.Y. "Probabilistic slope stability analysis of a $300 \mathrm{~m}$ high embankment dam", Conference Proceeding Paper of Geo-Risk: Risk Assessment and Management, GSP 224 (2011).

11. Cho, S.E. "Probabilistic assessment of slope stability that considers the spatial variability of soil properties", Journal of Geotechnical and Geoenvironmental Engineering (ASCE), 136(7), pp. 975-985 (2010).

12. Wang, Y., Cao, Z. and Au, S.K. "Practical reliability analysis of slope stability by advanced Monte-Carlo simulations in a spreadsheet", Canadian Geotechnical Journal, 48(1), pp. 162-172 (2011).

13. Jiang, S.H., Li, D.Q., Cao, Z.J., Zhou, C.B. and Phoon, K.K. "Efficient system reliability analysis of slope stability in spatially variable soils using Monte Carlo simulation", Journal of Geotechnical and Geoenvironmental Engineering (ASCE), 141(2), pp. 04014096-1-13 (2015).

14. Li, D.Q., Qi, X.H., Zhou, C.B. and Phoon, K.K. "Effect of spatially variable shear strength parameters with linearly increasing mean trend on reliability of infinite slopes", Structural Safety, 49, pp. 45-55 (2014).

15. Li, D.Q., Jiang, S.H., Cao, Z.J., Zhou, C.B. and Zhang, L.M. "A multiple response-surface method for slope reliability analysis considering spatial variability of soil properties", Engineering Geology, 187, pp. 6072 (2015).

16. Griffiths, D. and Fenton, G. "Probabilistic slope stability analysis by finite elements", Journal of Geotechnical and Geoenvironmental Engineering (ASCE), 130(5), pp. 507-518 (2004).

17. Griffiths, D.V., Huang, J. and Fenton, G.A. "Influence of spatial variability on slope reliability using 2-D random fields", Journal of Geotechnical and Geoenvironmental Engineering (ASCE), 132(11), pp. 14441454 (2009).

18. Xu, B., and Low, B.K. "Probabilistic stability analyses of embankments based on finite-element method", Journal of Geotechnical and Geoenvironmental Engineering (ASCE), 132(11), pp. 1444-1454 (2006).

19. Johari, A. and Javadi, A.A. "Reliability assessment of infinite slope stability using the jointly distributed random variables method", Scientia Iranica, 19(3), pp. 423-429 (2012).

20. Huang, J., Griffiths, D.V. and Fenton, G.A. "System reliability of slopes by RFEM", Soils and Foundations, 50(3), pp. 343-353 (2010).

21. Jiang, S.H., Li, D.Q., Zhang, L.M. and Zhou, C.B. "Slope reliability analysis considering spatially variable shear strength parameters using a non-intrusive stochastic finite element method", Engineering Geology, 168, pp. 120-168 (2014).

22. Vanmarcke, E.H. "Probabilistic modeling of soil profiles", Journal of Geotechnical and Geoenvironmental Engineering (ASCE), 103(11), pp. 147-166 (1977).

23. Jamshidi Chenari, R., Zhalehjoo, N. and Karimian, A. "Estimation on bearing capacity of shallow foundations in heterogeneous deposits using analytical and numerical methods", Scientia Iranica, Transactions A, Civil Engineering, 21(3), pp. 505-515 (2014).

24. Phoon, K.K. and Kulhawy, F.H. "Characterization of geotechnical variability", Canadian Geotechnical Journal, 36(4), pp. 612-624 (1999).

25. DeGroot, D.J. and Baecher, G.B. "Estimating autocovariance of in-situ soil properties", Journal of Geotechnical and Geoenvironmental Engineering (ASCE), 119(1), pp. 147-166 (1993).

26. Vanmarcke, E.H., Random Fields: Analysis and Synthesis, Cambridge, Massachusetts: The MIT Press (1983).

27. Fenton, G.A. and Griffiths, D.V. "Statistics of block conductivity through a simple bounded stochastic medium", Water Resource Research, 29(6), pp. 18251830 (1993).

28. Li, K.S. and Lumb, P. "Probabilistic design of slopes", Canadian Geotechnical Journal, 24(4), pp. 520-531 (1987).

29. Mostyn, G.R. and Soo, S. "The effect of autocorrelation on probability of failure of slopes", Proceedings of 6th Australian - New Zealand Conference on Geomechanics: Geotechnical Risk, pp. 542-546 (1992).

30. Low, B.K. and Tang, W.H. "Reliability analysis of reinforced embankments on soft grounds", Canadian Geotechnical Journal, 34(5), pp. 672-685 (1997a).

31. Low, B.K. and Tang, W.H. "Efficient reliability evaluation using spreadsheet", Journal of Geotechnical and Geoenvironmental Engineering (ASCE), 123(7), pp. 749-752 (1997b).

32. El-Ramly, H., Morgerstern, N.R. and Cruden, D.M. "Probabilistic slope stability analysis for practice", Canadian Geotechnical Journal, 39(3), pp. 665-683 (2002).

33. Low, B.K. "Practical probabilistic slope stability analysis", Proceedings of Soil and Rock America, 12th PanAmerican Conference on Soil Mechanics and Geotechnical Engineering and 39th U.S. Rock Mechanics Sym- 
posium, MIT, Cambridge, Massachusetts, 2, pp. 27772784, Essen, Germany (2003).

34. Sivakumar Babu, G.L. and Mukesh, M.D. "Effect of soil variability on reliability of soil slopes", Geotechnique, 54(5), pp. 335-337 (2004).

35. Low, B.K., Lacasse, S. and Nadim, F. "Slope reliability analysis accounting for spatial variation", Georisk, 1(4), pp. 177-189 (2007).

36. Cho, S.E. "Effect of spatial variability of soil properties on slope stability", Engineering Geology, 92(3-4), pp. 97-109 (2007).

37. Slope/W. (2008). Retrieved from Theory manual of version 2007: www.geo-slope.com/downloads/2007. aspx.

38. Catalan, J.M. and Comell, C.A. "Earth slope reliability by a level-crossing method", Journal of Geotechnical and Geoenvironmental Engineering (ASCE), 102(6), pp. 691-604 (1976).

39. Alonso, E.E. "Risk analysis of slopes and its application to slopes in Canadian sensitive clays", Geotechnique, 26, pp. 453-472 (1976).

40. Ching, J.Y., Phoon, K.K. and Hu, Y.G. "Efficient evaluation of reliability for slopes with circular slip surfaces using importance sampling", Journal of Geotechnical and Geoenvironmental Engineering (ASCE), 135(6), pp. 768-777 (2009).

41. Ching, J.Y., Phoon, K.K. and Hu, Y.G. "Observations on limit equilibrium based slope stability reliability problems with inclined weak seams", Journal of Engineering Mechanics (ASCE), 136(10), pp. 1220-1233 (2010).

42. Zhang, J., Huang, H.W. and Phoon, K.K. "Application of Kriging-Based response surface method for system reliability of soil slopes", Journal of Geotechnical and Geoenvironmental Engineering (ASCE), 139(4), pp. 651-655 (2013).

43. Chen, W.F. and Scawthorn, C.R., Limit Analysis and Limit Equilibrium Solution in Soil Mechanics, The Japanese Geotechnical Society, pp. 13-49 (1971).

44. FLAC 5.0 Reference Manual, Minneapolis: Itasca Consulting Inc. (2007).

45. Lee, I.K., White, W. and Ingles, O.G., Geotechnical Engineering, Pitman, London (1983).

46. Harr, M.E., Reliability Based Design in Civil Engineering, New York: McGraw Hill (1987).

47. Griffiths, D.V. and Fenton, G.A. "Bearing capacity of spatially random soil: the undrained clay Prandtl problem revisited", Geotechnique, 51(4), pp. 351-359 (2001).

48. El-Kadi, A.I. and Williams, S.A., Generating Two
Dimensional Fields of Autocorrelated, Normally Distributed Parameters By the Matrix Decomposition Technique, International Ground Water Modeling Center, pp. 530-532 (1999).

49. Fenton, G.A. and Griffiths, D.V. "The random finite element method (RFEM) in bearing capacity analyses", Probabilistic Methods in Geotechnical Engineering, CISM Course and Lectures, 491, pp. 295-31 (2007).

\section{Biographies}

Reza Jamshidi Chenari, PhD, PEng is an Associate Professor in the Group of Civil Engineering, University of Guilan. He is also a Professional Engineer in Iran. He received his BS (first class) and MS degrees from Sharif University of Technology and his PhD from Iran University of Science and Technology, Tehran, Iran, where his research focused on dynamic behavior of retaining walls backfilled with geotextile fiber reinforced sand. He conducted large cyclic triaxial tests and also shaking table experiments. Dr. Jamshidi's current research focuses on risk and reliability in geoengineering and geohazards, application of Random Numerical Methods (RNM) in different geotechnical problems including foundation settlement, shallow foundation bearing capacity, slope stability, consolidation of natural alluvial deposits, seismic amplification of alluviums, and in general sense, he now focuses on the effect of heterogeneity and anisotropy in deformation and strength properties of natural alluviums on the aforesaid problems. Dr. Jamshidi has authored or coauthored more than 40 scientific publications.

Masoud Zamanzadeh received his BS degree in Civil Engineering from Islamic Azad University of Marand in 2008 and MS degree in Geotechnical Engineering from University of Guilan in 2012. He fulfilled his master dissertation, focused on the "Uncertainty Assessment of Critical Excavation Depth of Vertical Unsupported Cuts in Undrained Clay Using Random Field Theorem", under the supervision of Dr. Reza Jamshidi Chenari in September 2012. As a geo-engineer, he is interested in computational geomechanics and the application of numerical modeling in geotechnical engineering practice. He has written scientific publications on risk and reliability in geotechnical engineering. His last contribution is the "Investigation into the effect of the inherent heterogeneity of soil on the long-term stability of earth slopes" accepted for publication in Sharif Civil Engineering Scientific Research Journal, Sharif University of Technology. 